\title{
Multiple Sign-Changing Solutions for Quasilinear Equations of Bounded Quasilinearity
}

\author{
Jiaquan $\mathrm{Liu}^{1}$, Xiangqing $\mathrm{Liu}^{2, *}$ and Zhi-Qiang Wang ${ }^{3,4}$ \\ ${ }^{1}$ LMAM, School of Mathematical Science, Peking University, Beijing 100871, China \\ 2 Department of Mathematics, Yunnan Normal University, Kunming, Yunnan 650500, \\ China \\ ${ }^{3}$ College of Mathematics and Informatics and Center for Applied Mathematics of \\ Fujian Province (FJNU), Fujian Normal University, Fuzhou, Fujian 350117, China \\ 4 Department of Mathematics and Statistics, Utah State University, Logan, \\ UT 84322, USA
}

Received 22 July 2020; Accepted (in revised version) 30 November 2020

Dedicated to Prof. Paul H. Rabinowitz with admiration on the occasion of his 80th birthday

Abstract. The existence of an infinite sequence of sign-changing solutions are proved for a class of quasilinear elliptic equations under suitable conditions on the quasilinear coefficients and the nonlinearity

$$
\begin{cases}\sum_{i, j=1}^{N}\left(b_{i j}(u) D_{i j} u+\frac{1}{2} D_{z} b_{i j}(u) D_{i} u D_{j} u\right)+f(u)=0 & \text { in } \Omega, \\ u=0 & \text { on } \partial \Omega,\end{cases}
$$

where $\Omega \subset \mathbb{R}^{N}$ is a bounded domain with smooth boundary, and we use

$$
D_{i} u=\frac{\partial u}{\partial x_{i}}, \quad D_{i j} u=\frac{\partial^{2} u}{\partial x_{i} \partial x_{j}}, \quad \text { and } \quad D_{z} b_{i j}(z)=\frac{d}{d z} b_{i j}(z) \text {. }
$$

The main interest of this paper is for the case of bounded quasilinearity $b_{i j}$. The result is proved by an elliptic regularization method involving truncations of both $u$ and the gradient of $u$.

Key Words: Quasilinear elliptic equations, sign-changing solution, an elliptic regularization method.

AMS Subject Classifications: 35J60, 35J20

${ }^{*}$ Corresponding author. Email addresses: jiaquan@math.pku.edu.cn (J. Q. Liu), lxq8u8@163.com (X. Q. Liu), zhi-qiang. wang@usu.edu (Q. Z. Wang) 


\section{Introduction}

In this paper, we study the existence of sign-changing solutions for the following quasilinear elliptic equation

$$
\begin{cases}\sum_{i, j=1}^{N}\left(b_{i j}(u) D_{i j} u+\frac{1}{2} D_{z} b_{i j}(u) D_{i} u D_{j} u\right)+f(u)=0 & \text { in } \Omega, \\ u=0 & \text { on } \partial \Omega,\end{cases}
$$

where $\Omega \subset \mathbb{R}^{N}$ is a bounded domain with smooth boundary, and we use the notations

$$
D_{i} u=\frac{\partial u}{\partial x_{i}}, \quad D_{i j} u=\frac{\partial^{2} u}{\partial x_{i} \partial x_{j}}, \quad D_{z} b_{i j}(z)=\frac{d}{d z} b_{i j}(z) .
$$

We assume the following conditions on $b_{i j}$ and $f$. Denote the critical exponent by $2^{*}=$ $\frac{2 N}{N-2}$ for $N \geq 3$ and $2^{*}=+\infty$ for $N=1,2$.

$\left(b_{1}\right)$ Let $b_{i j}=b_{j i} \in C^{1,1}(\mathbb{R}, \mathbb{R})$ for $i, j=1, \cdots, N$, satisfy that there exist positive constants $b_{-}, b_{+}$such that

$$
b_{-}|\xi|^{2} \leq \sum_{i, j=1}^{N} b_{i j}(z) \xi_{i} \xi_{j} \leq b_{+}|\xi|^{2} \quad \text { for } z \in \mathbb{R}, \quad \xi=\left(\xi_{i}\right) \in \mathbb{R}^{N} .
$$

$\left(b_{2}\right)$ There exist constants $q>2, \delta>0$ such that

$$
\begin{aligned}
\delta|\xi|^{2} & \leq \sum_{i, j=1}^{N}\left(b_{i j}(z)+\frac{1}{2} z D_{z} b_{i j}(z)\right) \xi_{i} \xi_{j} \\
& \leq \frac{q}{2}\left(\sum_{i, j=1}^{N} b_{i j}(z) \xi_{i} \xi_{j}-\delta|\xi|^{2}\right) \quad \text { for } z \in \mathbb{R}, \quad \xi \in \mathbb{R}^{N} .
\end{aligned}
$$

$\left(b_{3}\right)$ There exists a positive constant $c$ such that

$$
\left|D_{z} b_{i j}(z)-D_{z} b_{i j}(w)\right| \leq c|z-w| \quad \text { for } z, w \in \mathbb{R} .
$$

$\left(b_{4}\right) b_{i j}(z)$ is even in $z$.

$\left(f_{1}\right)$ Let $f \in C(\mathbb{R}, \mathbb{R})$ satisfy that there exist constants $c>0$ and $r \in\left(2,2^{*}\right)$ such that

$$
|f(z)| \leq c\left(1+|z|^{r-1}\right) \quad \text { for } z \in \mathbb{R} .
$$


$\left(f_{2}\right)$ There exists a positive constant $c$ such that

$$
\frac{1}{q} z f(z)-F(z) \geq-c \quad \text { for } z \in \mathbb{R}
$$

where $F(z)=\int_{0}^{z} f(\tau) d \tau$.

$\left(f_{3}\right) \lim _{z \rightarrow 0} \frac{f(z)}{z}=0, \quad \lim _{|z| \rightarrow+\infty} \frac{f(z)}{z}=+\infty$.

$\left(f_{4}\right) f(z)$ is odd in $z$.

For simplicity of notations we use the same $c$ to denote some constants in the above conditions.

We are looking for weak solutions for Eq. (1.1), namely a function $u \in H_{0}^{1}(\Omega) \cap L^{\infty}(\Omega)$ satisfying

$$
\begin{aligned}
& \int_{\Omega}\left(\sum_{i, j=1}^{N} b_{i j}(u) D_{i} u D_{j} \varphi+\frac{1}{2} D_{z} b_{i j}(u) D_{i} u D_{j} u \varphi\right) d x \\
= & \int_{\Omega} f(u) \varphi d x \text { for } \varphi \in C_{0}^{\infty}(\Omega) .
\end{aligned}
$$

For semilinear case, this is in the setting of the classical superlinear problems that goes back to the celebrated paper of Ambrosetti and Rabinowitz [1] (see also the book [24]) in which infinitely many solutions were obtained using the symmetric mountain pass theorem. Later a sequence of sign-changing solutions were assured to exist in the setting $[4,13]$.

The following is the main result of this paper which establish the above mentioned results for the case of bounded quasilinearity.

Theorem 1.1. Assume $\left(b_{1}\right)-\left(b_{4}\right),\left(f_{1}\right)-\left(f_{4}\right)$. Then Eq. (1.1) has infinitely many sign-changing solutions.

Formally the problem has a variational structure, given by the functional

$$
I(u)=\frac{1}{2} \int_{\Omega} \sum_{i, j=1}^{N} b_{i j}(u) D_{i} u D_{j} u d x-\int_{\Omega} F(u) d x \quad \text { for } u \in H_{0}^{1}(\Omega) .
$$

The functional $I$ is continuous, but not differentiable on $H_{0}^{1}(\Omega)$. Historically there have been several approaches developed for handling this type of non-smooth variational problems. The critical point theory for nonsmooth functionals has been established, see $[2,3,8,10,11,15]$. The authors of the present paper developed a new approach in [16, $17,20,21]$, by introducing a $p$-Laplacian perturbation. The theory in the above papers were used in [16] to treat the existence of an infinite sequence of sign-changing solutions 
for a problem similar to Eq. (1.1) but having a quasilinear term $b_{i j}$ of power growth in $u$. For some technical reasons, the $p$-Laplacian perturbation approach does not work well for the case we consider here, namely the quasilinear term $b_{i j}$ is bounded. The existence of a nontrivial solution for (1.1) was given in $[2,8]$. The question on the existence of sign-changing solutions in particular on whether there exist an infinite sequence of sign-changing solutions in this case was left open in $[8,16]$.

In this paper we will use a different approach which was used in [22] where localized solutions for semiclasscial Schrödinger type equations were considered. We outline the approach here first. Instead of introducing a $p$-Laplacian perturbation, we truncate the quadratic form $\sum_{i, j=1}^{N} b_{i j}(u) D_{i} u D_{j} u$. Under suitable truncations, up to some level sets controlled by the truncation, the resulting problem share critical points with the original problem. More precisely, let $\mu>0$ be a parameter and define a family of functionals

$$
\begin{gathered}
I_{\mu}(u)=\frac{1}{2} \sigma \int_{\Omega}|\nabla u|^{2} d x+\frac{1}{2} \int_{\Omega} h_{\mu}\left(\sum_{i, j=1}^{N} \beta_{i j}(u) D_{i} u D_{j} u\right) d x \\
-\int_{\Omega} F(u) d x, \quad u \in H_{0}^{1}(\Omega),
\end{gathered}
$$

where $\sigma>0$ is fixed satisfying

$$
0<\sigma<\min \left\{\frac{2}{q} \delta, \frac{1}{2} b_{-}\right\}
$$

$h_{\mu}$ is a smooth function such that $h_{\mu}(t)=t$ for $t<\frac{1}{\mu}$ and $h_{\mu}^{\prime}(t)=0$ for $t \geq \frac{T}{\mu}$, with $T>1$ being set as $T=2 e^{\frac{6 b_{+}}{\sigma}}+1$ (see Section 2 for details), and $\beta_{i j}(z)=b_{i j}(z)-\sigma \delta_{i j}$, $i, j=1, \cdots, N$.

We will verify that $I_{\mu}$ is a $C^{1}$-functional on $H_{0}^{1}(\Omega)$ and satisfies the Palais-Smale condition. The corresponding Euler-Lagrange equation for $I_{\mu}$ is

$$
\begin{aligned}
& \sigma \int_{\Omega} \nabla u \nabla \varphi d x+\int_{\Omega} h_{\mu}^{\prime}\left(\sum_{i, j=1}^{N} \beta_{i j}(u) D_{i} u D_{j} u\right) \sum_{i, j=1}^{N}\left(\beta_{i j}(u) D_{i} u D_{j} \varphi\right. \\
& \left.\quad+\frac{1}{2} D_{z} b_{i j}(z) D_{i} u D_{j} u \varphi\right) d x \\
& =\int_{\Omega} f(u) \varphi d x
\end{aligned}
$$

for $\varphi \in H_{0}^{1}(\Omega)$. Now assume $u$ is a solution of (1.5) (that is a critical point of (1.3)). Since the modification of the functional involves point-wisely the first order partial derivatives we will need some $C^{1}$-apriori estimates on the critical points of $I_{\mu}$ under energy bounds. If $u \in C^{1}(\bar{\Omega})$ and satisfies

$$
\sum_{i, j=1}^{N} \beta_{i j}(u) D_{i} u D_{j} u<\frac{1}{\mu} \quad \text { for } x \in \bar{\Omega},
$$


then $u$ will be a solution of the original equation (1.1). Note that no limit process $\mu \rightarrow 0$ is needed for the existence of critical point of the original problem. By taking smaller $\mu$ we obtain more solutions of the original problem. This is the main idea of the approach. In order to obtain sign-changing critical points of the functional $I_{\mu}$ we shall apply the method of invariant sets for descending flow, as developed in $[6,18,19]$. The idea of using invariant sets for descending flow for studying sign-changing solutions goes back to some earlier work for semilinear elliptic boundary value problems, such as in $[5,7,9,23]$ for the existence of sign-changing solutions and $[4,13]$ for an infinite sequence of signchanging solutions.

Throughout the paper, $c$ denotes various positive constants, $c_{\mu}$ denotes constants depending on $\mu$. The paper is organized as follows. In Lemma 2.1 we define the truncation function $h_{\mu}$. In Lemma 2.2 and Lemma 2.3 we prove the functional $I_{\mu}$ is a $C^{1}$-functional on $H_{0}^{1}(\Omega)$ and satisfies the Palais-Smale condition. Lemma 2.4 to Lemma 2.8 and Proposition 2.1 are devoted to constructing critical values of the functional $I_{\mu}$ by the method of invariant sets for descending flow. In Proposition 2.2 we prove the regularity result as the gradient estimate (1.6), which completes the proof of Theorem 1.1 consequently.

\section{Proof of Theorem 1.1}

We start with giving the definition of the function $h_{\mu}$ used in the truncation for the functional $I$ to get $I_{\mu}$.

Lemma 2.1. Given $\varepsilon>0$ there exists a smooth function $h \in C^{\infty}([0, \infty),[0, \infty))$ such that

(1) $h(t)=t$ for $t \leq 1$.

(2) $h^{\prime}(t)=1$ for $t \leq 1, h^{\prime}(t)=0$ for $t \geq T_{\varepsilon}=2 e^{\frac{1}{\varepsilon}}+1 ; h^{\prime}(t) \in[0,1], h^{\prime}(t)$ is decreasing in $t$, hence $h(t) \geq t h^{\prime}(t)$.

(3) $\left|h^{\prime \prime}(t)\right| t \leq \varepsilon$.

Proof. Choose a smooth function $\psi \in C^{\infty}(\mathbb{R},[0,1])$ such that $\psi(t)=1$ for $2 \leq t \leq 2 e^{\frac{1}{\varepsilon}}=$ $T_{\varepsilon}-1 ; \psi(t)=0$ for $t \leq 1$ or $t \geq T_{\varepsilon}$. We have

$$
M=\int_{\mathbb{R}} \frac{\psi(t)}{t} d t \geq \int_{2}^{T_{\varepsilon}-1} \frac{d t}{t}=\ln \frac{T_{\varepsilon}-1}{2}=\frac{1}{\varepsilon} .
$$

Now define

$$
q(t)=1-\frac{1}{M} \int_{-\infty}^{t} \frac{\psi(\tau)}{\tau} d \tau
$$

Then $0 \leq q(t) \leq 1, q(t)=1$ for $t \leq 1 ; q(t)=0$ for $t \geq T_{\varepsilon} ; q(t)$ is decreasing in $t$ and

$$
\left|q^{\prime}(t)\right| t=\frac{1}{M} \psi(t) \leq \frac{1}{M} \leq \varepsilon .
$$

Now $h(t)=\int_{0}^{t} q(\tau) d \tau$ is the desired function. 
In the future we choose and fix $\varepsilon$ and $T:=T_{\varepsilon}$ in the definition of $h$ above such that

$$
\varepsilon=\frac{\sigma}{6 b_{+}} \text {. }
$$

Let

$$
h_{\mu}(t)=\frac{1}{\mu} h(\mu t), \quad t \in[0, \infty), \quad \mu \in(0,1]
$$

We have

(1) $h_{\mu}(t)=t$ for $t \leq \frac{1}{\mu}, 0 \leq h_{\mu}(t) \leq \frac{T_{\varepsilon}}{\mu}$.

(2) $h_{\mu}^{\prime}(t)=h^{\prime}(\mu t) \in[0,1], h_{\mu}^{\prime}(t)=1$ for $t \leq \frac{1}{\mu}, h_{\mu}^{\prime}(t)=0$ for $t \geq \frac{T_{\varepsilon}}{\mu}, h_{\mu}^{\prime}$ is decreasing, hence $h_{\mu}(t) \geq t h_{\mu}^{\prime}(t)$.

(3) $\left|h_{\mu}^{\prime \prime}(t)\right| t=\left|h^{\prime \prime}(\mu t)\right| \mu t \leq \varepsilon$.

Lemma 2.2. The functional $I_{\mu}$ is differentiable and

$$
\begin{aligned}
& \left\langle D I_{\mu}(u), \varphi\right\rangle \\
= & \sigma \int_{\Omega} \nabla u \nabla \varphi d x+\int_{\Omega} h_{\mu}^{\prime}\left(\sum_{i, j=1}^{N} \beta_{i j}(u) D_{i} u D_{j} u\right) \\
& \quad \cdot \sum_{i, j=1}^{N}\left(\beta_{i j}(u) D_{i} u D_{j} \varphi+\frac{1}{2} D_{z} \beta_{i j}(u) D_{i} u D_{j} u \varphi\right) d x-\int_{\Omega} f(u) \varphi d x \quad \text { for } \varphi \in H_{0}^{1}(\Omega) .
\end{aligned}
$$

Proof. Let $H_{\mu}$ be the truncated quadratic term in $I_{\mu}$

$$
H_{\mu}(u)=\frac{1}{2} \int_{\Omega} h_{\mu}\left(\sum_{i, j=1}^{N} \beta_{i j}(u) D_{i} u D_{j} u\right) d x .
$$

The other two terms in $I_{\mu}$ are "harmless" to the smoothness. We compute the Gateaux derivative of $H_{\mu}$. The Gateaux derivative of $H_{\mu}$ at $u$ in the direction $\varphi$ is defined as

$$
\left\langle D_{G} H_{\mu}(u), \varphi\right\rangle=\lim _{t \rightarrow 0^{+}} \frac{1}{t}\left(H_{\mu}(u+t \varphi)-H_{\mu}(u)\right) .
$$

Denote $u_{t}=u+t \varphi, t \in[0,1]$. Then

$$
\begin{aligned}
& \frac{1}{t}\left(H_{\mu}(u+t \varphi)-H(u)\right) \\
= & \int_{\Omega} d x \frac{1}{t} \int_{0}^{t} \frac{d}{d t} \frac{1}{2} h_{\mu}\left(\sum_{i, j=1}^{N} \beta_{i j}\left(u_{t}\right) D_{i} u_{t} D_{j} u_{t}\right) d t \\
= & \int_{\Omega} d x \frac{1}{t} \int_{0}^{t} h_{\mu}^{\prime}\left(\sum_{i, j=1}^{N} \beta_{i j}\left(u_{t}\right) D_{i} u_{t} D_{j} u_{t}\right) \sum_{i, j=1}^{N}\left(\beta_{i j}\left(u_{t}\right) D_{i} u_{t} D_{j} \varphi+\frac{1}{2} D_{z} \beta_{i j}\left(u_{t}\right) D_{i} u_{t} D_{j} u_{t} \varphi\right) d t .
\end{aligned}
$$


Since

$$
\sum_{i, j=1}^{N} \beta_{i j}\left(u_{t}\right) D_{i} u_{t} D_{j} u_{t} \geq\left(b_{-}-\sigma\right)\left|\nabla u_{t}\right|^{2} \geq \frac{1}{2} b_{-}\left|\nabla u_{t}\right|^{2}
$$

we have

$$
h_{\mu}^{\prime}\left(\sum_{i, j=1}^{N} \beta_{i j}\left(u_{t}\right) D_{i} u_{t} D_{j} u_{t}\right)=0 \quad \text { for } \frac{1}{2} b_{-}\left|\nabla u_{t}\right|^{2} \geq \frac{T_{\varepsilon}}{\mu} .
$$

Hence

$$
\begin{aligned}
& \quad\left|\frac{1}{t} \int_{0}^{t} h_{\mu}^{\prime}\left(\sum_{i, j=1}^{N} \beta_{i j}\left(u_{t}\right) D_{i} u_{t} D_{j} u_{t}\right) \sum_{i, j=1}^{N}\left(\beta_{i j}\left(u_{t}\right) D_{i} u_{t} D_{j} \varphi+\frac{1}{2} D_{z} \beta_{i j}\left(u_{t}\right) D_{i} u_{t} D_{j} u_{t} \varphi\right) d t\right| \\
& \leq c_{\mu}(|\nabla \varphi|+|\varphi|) .
\end{aligned}
$$

By Lebesgue's dominated convergence theorem we have

$$
\begin{aligned}
& \left\langle D_{G} H_{\mu}(u), \varphi\right\rangle=\lim _{t \rightarrow 0^{+}} \frac{1}{t}\left(H_{\mu}(u+t \varphi)-H_{\mu}(u)\right) \\
& =\int_{\Omega} h_{\mu}^{\prime}\left(\sum_{i, j=1}^{N} \beta_{i j}(u) D_{i} u D_{j} u\right) \sum_{i, j=1}^{N}\left(\beta_{i j}(u) D_{i} u D_{j} \varphi+\frac{1}{2} D_{z} \beta_{i j}(u) D_{i} u D_{j} u \varphi\right) d x, \\
& \left\langle D_{G} H_{\mu}(u), \varphi\right\rangle \leq c_{\mu} \int_{\Omega}(|\nabla \varphi|+|\varphi|) d x \leq c_{\mu}\|\varphi\|, \quad \varphi \in H_{0}^{1}(\Omega) .
\end{aligned}
$$

The Gateaux derivative $D_{G} H_{\mu}(u)$ is a bounded linear functional on $H_{0}^{1}(\Omega)$. Moreover $D_{G} H_{\mu}(u)$ continuously depends on $u$ and there exists a positive constant $c_{\mu}$ such that

$$
\left\|D_{G} H_{\mu}(u)-D_{G} H_{\mu}(v)\right\| \leq c_{\mu}\|u-v\| \quad \text { for } u, v \in H_{0}^{1}(\Omega)
$$

In fact denoting $w_{t}=t u+(1-t) v, t \in[0,1]$ for $\varphi \in H_{0}^{1}(\Omega)$, we have

$$
\begin{aligned}
& \left\langle D_{G} H_{\mu}(u)-D_{G} H_{\mu}(v), \varphi\right\rangle \\
= & \int_{\Omega} h_{\mu}^{\prime}\left(\sum_{i, j=1}^{N} \beta_{i j}(u) D_{i} u D_{j} u\right)\left(\sum_{i, j=1} \beta_{i j}(u) D_{i} u D_{j} \varphi+\frac{1}{2} D_{z} \beta_{i j}(u) D_{i} u D_{j} u \varphi\right) d x \\
& \quad-\int_{\Omega} h_{\mu}^{\prime}\left(\sum_{i, j=1}^{N} \beta_{i j}(v) D_{i} v D_{j} v\right)\left(\sum_{i, j=1}^{N} \beta_{i j}(v) D_{i} v D_{j} \varphi+\frac{1}{2} D_{z} \beta_{i j}(v) D_{i} v D_{j} v \varphi\right) d x \\
= & \int_{\Omega} d x \int_{0}^{1} d_{t}\left\{h_{\mu}^{\prime}\left(\sum_{i, j=1}^{N} \beta_{i j}\left(w_{t}\right) D_{i} w_{t} D_{j} w_{t}\right)\left(\sum_{i, j=1}^{N} \beta_{i j}\left(w_{t}\right) D_{i} w_{t} D_{j} \varphi+\frac{1}{2} D_{z} \beta_{i j}\left(w_{t}\right) D_{i} w_{t} D_{j} w_{t} \varphi\right)\right\}
\end{aligned}
$$




$$
\begin{aligned}
& =\int_{\Omega} d x \int_{0}^{1} h_{\mu}^{\prime}\left(\sum_{i, j=1}^{N} \beta_{i j}\left(w_{t}\right) D_{i} w_{t} D_{j} w_{t}\right) \sum_{i, j=1}^{N}\left(\beta_{i j}\left(w_{t}\right) D_{i}(u-v) D_{j} \varphi\right. \\
& \left.\quad+D_{z} \beta_{i j}\left(w_{t}\right)(u-v) D_{i} w_{t} D_{j} \varphi+D_{z} \beta_{i j}\left(w_{t}\right) D_{i}(u-v) D_{j} w_{t} \varphi\right) d t \\
& \quad+\int_{\Omega} d x \int_{0}^{1} h_{\mu}^{\prime}\left(\sum_{i, j=1}^{N} \beta_{i j}\left(w_{t}\right) D_{i} w_{t} D_{j} w_{t}\right) \sum_{i, j=1}^{N} \frac{1}{2} D_{i} w_{t} D_{j} w_{t} \varphi d_{t} D_{z} \beta_{i j}\left(w_{t}\right) \\
& \quad+\int_{\Omega} d x \int_{0}^{1} h_{\mu}^{\prime \prime}\left(\sum_{i, j=1}^{N} \beta_{i j}\left(w_{t}\right) D_{i} w_{t} D_{j} w_{t}\right)\left(\sum_{i, j=1}^{N} 2 \beta_{i j}\left(w_{t}\right) D_{i}(u-v) D_{j} w_{t}\right. \\
& \left.\quad+D_{z} \beta_{i j}\left(w_{t}\right)(u-v) D_{i} w_{t} D_{j} w_{t}\right) \cdot\left(\sum_{i, j=1}^{N} \beta_{i j}\left(w_{t}\right) D_{i} w_{t} D_{j} \varphi+\frac{1}{2} D_{z} \beta_{i j}\left(w_{t}\right) D_{i} w_{t} D_{j} w_{t} \varphi\right) d t \\
& \leq c_{\mu} \int_{\Omega}(|\nabla(u-v)|+|u-v|)(|\nabla \varphi|+|\varphi|) d x \\
& \leq c_{\mu}\|u-v\|\|\varphi\| .
\end{aligned}
$$

In the above we have used the fact that

$$
\begin{aligned}
& \left|d_{t} D_{z} \beta_{i j}\left(w_{t}\right)\right| \leq c\left|d w_{t}\right| \leq c|u-v||d t| \\
& h_{\mu}^{\prime}\left(\sum_{i, j=1}^{N} \beta_{i j}\left(w_{t}\right) D_{i} w_{t} D_{j} w_{t}\right)=h_{\mu}^{\prime \prime}\left(\sum_{i, j=1}^{N} \beta_{i j}\left(w_{t}\right) D_{i} w_{t} D_{j} w_{t}\right) \\
& \quad=0 \text { for } \frac{1}{2} b_{-}\left|\nabla w_{t}\right|^{2} \geq \frac{T_{\varepsilon}}{\mu} .
\end{aligned}
$$

Since the Gateaux derivative $D_{G} H_{\mu}(u)$ is a bounded linear functional on $H_{0}^{1}(\Omega)$ and continuously depends on $u, H_{\mu}(u)$ is Frechet differentiable and the Frechet derivative $D H_{\mu}(u)=D_{G} H_{\mu}(u)$. Finally

$$
\begin{aligned}
& \left\langle D I_{\mu}(u), \varphi\right\rangle \\
= & \sigma \int_{\Omega} \nabla u \nabla \varphi d x+\left\langle D H_{\mu}(u), \varphi\right\rangle-\int_{\Omega} f(u) \varphi d x \\
= & \sigma \int_{\Omega} \nabla u \nabla \varphi d x+\int_{\Omega} h_{\mu}^{\prime}\left(\sum_{i, j=1}^{N} \beta_{i j}(u) D_{i} u D_{j} u\right) \\
& \cdot \sum_{i, j=1}^{N}\left(\beta_{i j}(u) D_{i} u D_{j} \varphi+\frac{1}{2} D_{z} \beta_{i j}(u) D_{i} u D_{j} u \varphi\right) d x-\int_{\Omega} f(u) \varphi d x
\end{aligned}
$$

for $\varphi \in H_{0}^{1}(\Omega)$.

Lemma 2.3. $I_{\mu}$ satisfies the Palais-Smale condition. 
Proof. We have

$$
\begin{aligned}
& I_{\mu}(u)-\frac{1}{q}\left\langle D I_{\mu}(u), u\right\rangle \\
& =\left(\frac{1}{2}-\frac{1}{q}\right) \sigma \int_{\Omega}|\nabla u|^{2} d x+\int_{\Omega}\left\{\frac{1}{2} h_{\mu}\left(\sum_{i, j=1}^{N} \beta_{i j}(u) D_{i} u D_{j} u\right)-\frac{1}{q} h_{\mu}^{\prime}\left(\sum_{i, j=1}^{N} \beta_{i j}(u) D_{i} u D_{j} u\right)\right. \\
& \left.\quad \cdot \sum_{i, j=1}^{N}\left(\beta_{i j}(u)+\frac{1}{2} u D_{z} \beta_{i j}(u)\right) D_{i} u D_{j} u\right\} d x+\int_{\Omega}\left(\frac{1}{q} u f(u)-F(u)\right) d x \\
& \geq\left(\frac{1}{2}-\frac{1}{q}\right) \sigma \int_{\Omega}|\nabla u|^{2} d x+\int_{\Omega}\left\{\frac{1}{2} h_{\mu}\left(\sum_{i, j=1}^{N} \beta_{i j}(u) D_{i} u D_{j} u\right)-\frac{1}{q} h_{\mu}^{\prime}\left(\sum_{i, j=1}^{N} \beta_{i j}(u) D_{i} u D_{j} u\right)\right. \\
& \left.\quad \cdot\left(\frac{q}{2} \sum_{i, j=1}^{N} \beta_{i j}(u) D_{i} u D_{j} u+\left(\frac{q}{2}(\sigma-\delta)-\sigma\right)|\nabla u|^{2}\right)\right\} d x+\int_{\Omega}\left(\frac{1}{q} u f(u)-F(u)\right) d x \\
& \geq\left(\frac{1}{2}-\frac{1}{q}\right) \sigma \int_{\Omega}|\nabla u|^{2} d x+\frac{1}{2} \int_{\Omega}\left\{h_{\mu}\left(\sum_{i, j=1}^{N} \beta_{i j}(u) D_{i} u D_{j} u\right)-h_{\mu}^{\prime}\left(\sum_{i, j=1}^{N} \beta_{i j}(u) D_{i} u D_{j} u\right)\right. \\
& \left.\quad \cdot \sum_{i, j=1}^{N} \beta_{i j}(u) D_{i} u D_{j} u\right\} d x-c \\
& \geq\left(\frac{1}{2}-\frac{1}{q}\right) \sigma \int_{\Omega}|\nabla u|^{2} d x-c .
\end{aligned}
$$

In the above we have used the fact that

$$
\sigma<\frac{2}{q} \delta, \quad h^{\prime}(t) \geq 0, \quad \frac{1}{q} z f(z)-F(z) \geq-c \quad \text { and } \quad h_{\mu}(t) \geq t h_{\mu}^{\prime}(t) .
$$

Let $\left\{u_{n}\right\} \subset H_{0}^{1}(\Omega)$ be a Palais-Smale sequence of $I_{\mu}$. By (2.3), $u_{n}$ is bounded in $H_{0}^{1}(\Omega)$. Assume $u_{n} \rightarrow u$ in $H_{0}^{1}(\Omega), u_{n} \rightarrow u$ in $L^{s}(\Omega)\left(1 \leq s<2^{*}\right), u_{n}(x) \rightarrow u(x)$ a.e. $x \in \Omega$. We have

$$
\begin{aligned}
o(1)= & \left\langle D I_{\mu}\left(u_{n}\right)-D I_{\mu}\left(u_{m}\right), u_{n}-u_{m}\right\rangle \\
= & \sigma \int_{\Omega}\left|\nabla\left(u_{n}-u_{m}\right)\right|^{2} d x+\left\langle D H_{\mu}\left(u_{n}\right)-D H_{\mu}\left(u_{m}\right), u_{n}-u_{m}\right\rangle \\
& \quad-\int_{\Omega}\left(f\left(u_{n}\right)-f\left(u_{m}\right)\right)\left(u_{n}-u_{m}\right) d x \\
= & \sigma \int_{\Omega}\left|\nabla\left(u_{n}-u_{m}\right)\right|^{2} d x+\left\langle D H_{\mu}\left(u_{n}\right)-D H_{\mu}\left(u_{m}\right), u_{n}-u_{m}\right\rangle+o(1) .
\end{aligned}
$$

We estimate the term $\left\langle D H_{\mu}\left(u_{n}\right)-D H_{\mu}\left(u_{m}\right), u_{n}-u_{m}\right\rangle$. Assume $u, v \in H_{0}^{1}(\Omega)$. Denote 
$w_{t}=t u+(1-t) v, t \in[0,1]$. We have

$$
\begin{aligned}
& \left\langle D H_{\mu}(u)-D H_{\mu}(v), u-v\right\rangle \\
& =\int_{\Omega} h_{\mu}^{\prime}\left(\sum_{i, j=1}^{N} \beta_{i j}(u) D_{i} u D_{j} u\right) \sum_{i, j=1}^{N}\left(\beta_{i j}(u) D_{i} u D_{j}(u-v)+\frac{1}{2} D_{z} \beta_{i j}(u) D_{i} u D_{j} u(u-v)\right) d x \\
& -\int_{\Omega} h_{\mu}^{\prime}\left(\sum_{i, j=1}^{N} \beta_{i j}(v) D_{i} v D_{j} v\right) \sum_{i, j=1}^{N}\left(\beta_{i j}(v) D_{i} v D_{j}(u-v)+\frac{1}{2} D_{z} \beta_{i j}(v) D_{i} v D_{j} v(u-v)\right) d x \\
& =\int_{\Omega} d x \int_{0}^{1} d_{t}\left\{h _ { \mu } ^ { \prime } ( \sum _ { i , j = 1 } ^ { N } \beta _ { i j } ( w _ { t } ) D _ { i } w _ { t } D _ { j } w _ { t } ) \sum _ { i , j = 1 } ^ { N } \left(\beta_{i j}\left(w_{t}\right) D_{i} w_{t} D_{j}(u-v)\right.\right. \\
& \left.\left.+\frac{1}{2} D_{z} \beta_{i j}\left(w_{t}\right) D_{i} w_{t} D_{j} w_{t}(u-v)\right)\right\} \\
& =\int_{\Omega} d x \int_{0}^{1} h_{\mu}^{\prime}\left(\sum_{i, j=1}^{N} \beta_{i j}\left(w_{t}\right) D_{i} w_{t} D_{j} w_{t}\right) \sum_{i, j=1}^{N}\left(\beta_{i j}\left(w_{t}\right) D_{i}(u-v) D_{j}(u-v)\right. \\
& \left.+2 D_{z} \beta_{i j}\left(w_{t}\right) D_{i} w_{t}(u-v) D_{j}(u-v)\right) d t \\
& +\int_{\Omega} d x \int_{0}^{1} h_{\mu}^{\prime}\left(\sum_{i, j=1}^{N} \beta_{i j}\left(w_{t}\right) D_{i} w_{t} D_{j} w_{t}\right) \sum_{i, j=1}^{N} \frac{1}{2} D_{i} w_{t} D_{j} w_{t}(u-v) d_{t} D_{z} \beta_{i j}\left(w_{t}\right) \\
& +\int_{\Omega} d x \int_{0}^{1} 2 h_{\mu}^{\prime \prime}\left(\sum_{i, j=1}^{N} \beta_{i j}\left(w_{t}\right) D_{i} w_{t} D_{j} w_{t}\right)\left(\sum _ { i , j = 1 } ^ { N } \left(\beta_{i j}\left(w_{t}\right) D_{i} w_{t} D_{j}(u-v)\right.\right. \\
& \left.\left.+\frac{1}{2} D_{z} \beta_{i j}\left(w_{t}\right) D_{i} w_{t} D_{j} w_{t}(u-v)\right)\right)^{2} d t \\
& \geq-c_{\mu} \int_{\Omega}(|\nabla(u-v)+| u-v \mid)|u-v| d x+\int_{\Omega} d x \int_{0}^{1} 2 h_{\mu}^{\prime \prime}\left(\sum_{i, j=1}^{N} \beta_{i j}\left(w_{t}\right) D_{i} w_{t} D_{j} w_{t}\right) \\
& \cdot \sum_{i, j=1}^{N} \beta_{i j}\left(w_{t}\right) D_{i} w_{t} D_{j} w_{t} \cdot \sum_{i, j=1}^{N} \beta_{i j}\left(w_{t}\right) D_{i}(u-v) D_{j}(u-v) d t \\
& \geq-c_{\mu} \int_{\Omega}(|\nabla(u-v)+| u-v \mid)|u-v| d x-2 \varepsilon b_{+} \int_{\Omega}|\nabla(u-v)|^{2} d x \\
& \geq-c_{\mu}^{*} \int_{\Omega}(u-v)^{2} d x-3 \varepsilon b_{+} \int_{\Omega}|\nabla(u-v)|^{2} d x .
\end{aligned}
$$

Here $\varepsilon$ is from (2.1) so that $\varepsilon b_{+}=\frac{\sigma}{6}$. It follows (2.4), (2.5) that

$$
\begin{aligned}
o(1) & \geq \sigma\left\|u_{n}-u_{m}\right\|^{2}-3 \varepsilon b_{+}\left\|u_{n}-u_{m}\right\|^{2}-c_{\mu}^{*}\left|u_{n}-u_{m}\right|_{L^{2}(\Omega)}^{2}+o(1) \\
& \geq \frac{1}{2} \sigma\left\|u_{n}-u_{m}\right\|^{2}+o(1)
\end{aligned}
$$

$u_{n}$ is a Cauchy sequence, hence a convergent sequence in $H_{0}^{1}(\Omega)$. 
From the proof of Lemma 2.2 and Lemma 2.3 (see (2.2), (2.5)), we have the following lemma, which is useful in our proofs later.

Lemma 2.4. There exist positive constants $c_{\mu}$ and $c_{\mu}^{*}$ such that

(1) $\left\|D H_{\mu}(u)-D H_{\mu}(v)\right\| \leq c_{\mu}\|u-v\|$.

(2) $\left\langle D H_{\mu}(u)-D H_{\mu}(v), u-v\right\rangle \geq-\frac{\sigma}{2}\|u-v\|^{2}-c_{\mu}^{*}|u-v|_{L^{2}(\Omega)}^{2}$.

We construct multiple solutions of the approximate problem (1.5), in particular multiple sign-changing solutions by the method of invariant sets for descending flow. The abstract framework is established in $[16,18,19,23]$ generalizing the classical mountain pass theorems without the setting of invariant sets [1,24].

Proposition 2.1. Let $X$ be a Banach space, $f$ be an even $C^{1}$-functional on $X$ and satisfy the Palais-Smale condition. Let $P, Q$ be open convex subsets of $X, Q=-P, W=P \cup Q, W \neq \varnothing$. Assume there exists an odd map $A: X \rightarrow X$ satisfying

$\left(A_{1}\right)$ Given $c_{0}, b_{0}>0$ there exists $b=b\left(c_{0}, b_{0}\right)$ such that if $\|D f(x)\| \geq b_{0},|f(x)| \leq c_{0}$ then

$$
\langle D f(x), x-A x\rangle \geq b\|x-A x\|>0 .
$$

$\left(A_{2}\right) A(\partial P) \subset P, A(\partial Q) \subset Q$.

Let $E$ be a finite-dimensional subspace of $X$ and $B$ be a ball in E centered at the origin. Define

$$
\begin{aligned}
& c=\inf _{\varphi \in \Gamma} \sup _{u \in \varphi(B) \backslash W} f(u), \\
& \Gamma=\left\{\varphi \mid \varphi \in C(B, X), \varphi \text { is odd, }\left.\varphi\right|_{\partial B}=I d\right\} .
\end{aligned}
$$

Assume

(C) $c>0 \geq \sup _{x \in \partial B} f(x)$.

Then $c$ is a critical value of $f$ and

$$
K_{c}^{*}=\{x \mid x \in X \backslash W, D f(x)=0, f(x)=c\} \neq \varnothing .
$$

In the following we verify that $I_{\mu}$ satisfies all the assumptions of Proposition 2.1 for a suitable operator $A$ and subsets $P, Q$ and $B$. In Lemma 2.2 and Lemma 2.3 we have proved that $I_{\mu}$ is a $C^{1}$-functional on $H_{0}^{1}(\Omega)$ and satisfies the Palais-Smale condition. Now we define the operator $A$ and the subsets $P, Q$ and verify the assumptions $\left(A_{1}\right),\left(A_{2}\right)$. 
Definition 2.1. Given $u \in H_{0}^{1}(\Omega)$, define $v=A u \in H_{0}^{1}(\Omega)$ by the following equation

$$
\begin{aligned}
\left\langle D J_{\mu}(v), \varphi\right\rangle & =\sigma \int_{\Omega} \nabla v \nabla \varphi d x+\left\langle D H_{\mu}(v), \varphi\right\rangle+c_{\mu}^{*} \int_{\Omega} v \varphi d x \\
& =\int_{\Omega} f(u) \varphi d x+c_{\mu}^{*} \int_{\Omega} u \varphi d x \quad \text { for } \varphi \in H_{0}^{1}(\Omega),
\end{aligned}
$$

where $c_{\mu}^{*}$ is the positive constant which appears in Lemma 2.4 and

$$
J_{\mu}(v)=\frac{1}{2} \sigma \int_{\Omega}|\nabla v|^{2} d x+H_{\mu}(v)+\frac{1}{2} c_{\mu}^{*} \int_{\Omega} v^{2} d x .
$$

Without loss of generality we assume

$$
\left(f(z)+c_{\mu}^{*} z\right) z \geq 0 \quad \text { for } z \in \mathbb{R} .
$$

Lemma 2.5. The operator $A$ is well-defined and continuous.

Proof. By Lemma 2.4 we have for $v, \bar{v} \in H_{0}^{1}(\Omega)$

$$
\begin{aligned}
& \left\langle D J_{\mu}(v)-D J_{\mu}(\bar{v}), v-\bar{v}\right\rangle \\
= & \sigma\|v-\bar{v}\|^{2}+\left\langle D H_{\mu}(v)-D H_{\mu}(\bar{v}), v-\bar{v}\right\rangle+c_{\mu}^{*}|v-v|_{L^{2}(\Omega)}^{2} \\
\geq & \sigma\|v-\bar{v}\|^{2}-\frac{\sigma}{2}\|v-\bar{v}\|^{2} \geq \frac{1}{2} \sigma\|v-\bar{v}\|^{2} .
\end{aligned}
$$

$D J_{\mu}(v)$ is a strongly monotone operator, hence given $u \in H_{0}^{1}(\Omega)$, Eq. (2.6) has a unique solution $v=A u \in H_{0}^{1}(\Omega)$. Moreover assume $u, \bar{u} \in H_{0}^{1}(\Omega), v=A u, \bar{v}=A \bar{u}$, then

$$
\begin{aligned}
& \frac{1}{2} \sigma\|v-\bar{v}\|^{2} \leq\left\langle D J_{\mu}(v)-D J_{\mu}(\bar{v}), v-\bar{v}\right\rangle \\
= & \int_{\Omega}(f(u)-f(\bar{u}))(v-\bar{v}) d x+c_{\mu}^{*} \int_{\Omega}(u-\bar{u})(v-\bar{v}) d x \\
\leq & |f(u)-f(\bar{u})|_{L^{\bar{r}}(\Omega)}|v-\bar{v}|_{L^{r}(\Omega)}+c_{\mu}^{*}|u-\bar{u}|_{L^{2}(\Omega)}|v-\bar{v}|_{L^{2}(\Omega)} \\
\leq & c_{\mu}\left(|f(u)-f(\bar{u})|_{L^{\bar{r}}(\Omega)}+|u-\bar{u}|_{L^{2}(\Omega)}\right)\|v-\bar{v}\|,
\end{aligned}
$$

where $\frac{1}{r}+\frac{1}{\bar{r}}=1$. Hence

$$
\|v-\bar{v}\| \leq c_{\mu}\left(|f(u)-f(\bar{u})|_{L^{\bar{r}}(\Omega)}+|u-\bar{u}|_{L^{2}(\Omega)}\right) \rightarrow 0 \quad \text { as } \bar{u} \rightarrow u \quad \text { in } H_{0}^{1}(\Omega) .
$$

We complete the proof.

Lemma 2.6. There exists a constant $c_{\mu}$ such that

$$
\left\|D I_{\mu}(u)\right\| \leq c_{\mu}\|u-A u\| .
$$

Moreover

$$
\left\langle D I_{\mu}(u), u-A u\right\rangle \geq \frac{1}{2} \sigma\|u-A u\|^{2} .
$$

Consequently the assumption $\left(A_{1}\right)$ holds. 
Proof. Assume $u \in H_{0}^{1}(\Omega), v=A u$. By the definition of the operator $A$,

$$
\begin{aligned}
& \sigma \int_{\Omega} \nabla v \nabla \varphi d x+\left\langle D H_{\mu}(v), \varphi\right\rangle+c_{\mu}^{*} \int_{\Omega} v \varphi d x \\
= & \int_{\Omega} f(u) \varphi d x+c_{\mu}^{*} \int_{\Omega} u \varphi d x \text { for } \varphi \in H_{0}^{1}(\Omega) .
\end{aligned}
$$

We have

$$
\begin{aligned}
& \left\langle D I_{\mu}(u), \varphi\right\rangle=\sigma \int_{\Omega} \nabla u \nabla \varphi d x+\left\langle D H_{\mu}(u), \varphi\right\rangle-\int_{\Omega} f(u) \varphi d x \\
= & \sigma \int_{\Omega} \nabla(u-v) \nabla \varphi d x+\left\langle D H_{\mu}(u)-D H_{\mu}(v), \varphi\right\rangle+c_{\mu}^{*} \int_{\Omega}(u-v) \varphi d x .
\end{aligned}
$$

By Lemma 2.4, we have

$$
\begin{gathered}
\left\|D I_{\mu}(u)\right\| \leq \sigma\|u-v\|+\left\|D H_{\mu}(u)-D H_{\mu}(v)\right\|+c_{\mu}\|u-v\| \leq c_{\mu}\|u-v\|, \\
\left\langle D I_{\mu}(u), u-v\right\rangle=\sigma\|u-v\|^{2}+\left\langle D H_{\mu}(u)-D H_{\mu}(v), u-v\right\rangle+c_{\mu}^{*}|u-v|_{L^{2}(\Omega)}^{2} \\
\geq \sigma\|u-v\|^{2}-3 \varepsilon b_{+}\|u-v\|^{2}=\frac{1}{2} \sigma\|u-v\|^{2} .
\end{gathered}
$$

Thus, we complete the proof.

We define the open convex sets $P$ and $Q$, and verify the assumption $\left(A_{2}\right)$ of Proposition 2.1.

Definition 2.2. Define the open convex sets $P$ and $Q$ as

$$
P=P_{v}=\left\{\left.u\left|u \in H_{0}^{1}(\Omega), \sigma S\right| u_{-}\right|_{L^{r}(\Omega)} ^{2}+c_{\mu}^{*}\left|u_{-}\right|_{L^{2}(\Omega)}^{2}<v^{2}\right\}, \quad Q=-P,
$$

where $u_{ \pm}=\max \{ \pm u, 0\}, c_{\mu}^{*}$ is the constant in Lemma $2.4, S=S_{p}(\Omega)$ is the Sobolev constant for the embedding $H_{0}^{1}(\Omega) \hookrightarrow L^{p}(\Omega), v>0$ is a small constant.

Lemma 2.7. There exists a positive constant $v_{0}$ such that for $v<v_{0}$, it holds that $A(\partial P) \subset P$, $A(\partial Q) \subset Q$.

Proof. Assume $u \in \partial Q, v=A u$. By the definitions

$$
\begin{aligned}
& \sigma S\left|u_{+}\right|_{L^{r}(\Omega)}^{2}+c_{\mu}^{*}\left|u_{+}\right|_{L^{2}(\Omega)}^{2}=v^{2} \\
& \sigma \int_{\Omega} \nabla v \nabla \varphi d x+\left\langle D H_{\mu}(v), \varphi\right\rangle+c_{\mu}^{*} \int_{\Omega} v \varphi d x \\
& \quad=\int_{\Omega} f(u) \varphi d x+c_{\mu}^{*} \int_{\Omega} u \varphi d x \text { for } \varphi \in H_{0}^{1}(\Omega) .
\end{aligned}
$$

Choose $\varphi=v_{+}$as test function in (2.8b). Since

$$
\left\langle D H_{\mu}(v), v_{+}\right\rangle=\left\langle D H_{\mu}\left(v_{+}\right), v_{+}\right\rangle \geq 0,
$$


the left hand side of $(2.8 b)$

$$
\begin{aligned}
\text { LHS } & \geq \sigma \int_{\Omega}\left|\nabla v_{+}\right|^{2} d x+c_{\mu}^{*} \int_{\Omega} v_{+}^{2} d x \\
& \geq \sigma S\left|v_{+}\right|_{L^{r}(\Omega)}^{2}+c_{\mu}^{*}\left|v_{+}\right|_{L^{2}(\Omega)}^{2} .
\end{aligned}
$$

For any $\tau>0$, by the assumptions $\left(f_{1}\right),\left(f_{3}\right)$, there exists $c_{\tau}>0$ such that

$$
|f(z)| \leq \tau|z|+c_{\tau}|z|^{r-1} \text {. }
$$

The right hand side of $(2.8 \mathrm{~b})$,

$$
\begin{aligned}
\mathrm{RHS} & =\int_{\Omega} f(u) v_{+} d x+c_{\mu}^{*} \int_{\Omega} u v_{+} d x \\
& \leq \int_{\Omega} f\left(u_{+}\right) v_{+} d x+c_{\mu}^{*} \int_{\Omega} u_{+} v_{+} d x \\
& \leq \int_{\Omega}\left(\tau u_{+}+c_{\tau} u_{+}^{r-1}\right) v_{+} d x+c_{\mu}^{*} \int_{\Omega} u_{+} v_{+} d x \\
& \leq\left(\tau|\Omega|^{1-\frac{2}{r}}+c_{\tau}\left|u_{+}\right|_{L^{r}(\Omega)}^{r-2}\right)\left|u_{+}\right|_{L^{r}(\Omega)}\left|v_{+}\right|_{L^{r}(\Omega)}+c_{\mu}^{*}\left|u_{+}\right|_{L^{2}(\Omega)}\left|v_{+}\right|_{L^{2}(\Omega)} \\
& \leq\left(\tau|\Omega|^{1-\frac{2}{r}}+c_{\tau}\left(\frac{v^{2}}{\sigma S}\right)^{\frac{r-2}{2}}\right)\left|u_{+}\right|_{L^{r}(\Omega)}\left|v_{+}\right|_{L^{r}(\Omega)}+c_{\mu}^{*}\left|u_{+}\right|_{L^{2}(\Omega)}\left|v_{+}\right|_{L^{2}(\Omega)} \\
& \leq \frac{1}{2} \sigma S\left|u_{+}\right|_{L^{r}(\Omega)}\left|v_{+}\right|_{L^{r}(\Omega)}+c_{\mu}^{*}\left|u_{+}\right|_{L^{2}(\Omega)}\left|v_{+}\right|_{L^{2}(\Omega)},
\end{aligned}
$$

provided we choose $\tau$ and $v_{0}$ such that

$$
\tau|\Omega|^{1-\frac{2}{r}} \leq \frac{1}{4} \sigma S \quad \text { and } \quad c_{\tau}\left(\frac{v_{0}^{2}}{\sigma S}\right)^{\frac{r-2}{2}} \leq \frac{1}{4} \sigma S, \quad v \leq v_{0}
$$

By (2.9) and (2.10), for $0<v \leq v_{0}$ we have

$$
\sigma S\left|v_{+}\right|_{L^{r}(\Omega)}^{2}+c_{\mu}^{*}\left|v_{+}\right|_{L^{2}(\Omega)}^{2} \leq \frac{1}{2} \sigma S\left|u_{+}\right|_{L^{r}(\Omega)}^{2}+c_{\mu}^{*}\left|u_{+}\right|_{L^{2}(\Omega)}^{2}<v^{2},
$$

hence $v=A u \in Q$ and $A(\partial Q) \subset Q$. Similarly $A(\partial P) \subset P$.

We apply the abstract theorem (Proposition 2.1) to define a sequence of critical values of the functional $I_{\mu}$, and estimate the bound of the critical values.

Definition 2.3. Let $0<\lambda_{1}<\lambda_{2} \leq \cdots$ be the eigenvalues of the Laplacian operator $-\Delta$ in $H_{0}^{1}(\Omega) \cap H^{2}(\Omega), e_{1}, e_{2}, \cdots$, be the corresponding eigenfunctions. Denote

$$
E_{l}=\operatorname{span}\left\{e_{1}, e_{2}, \cdots, e_{l}\right\}, \quad B_{l}=\left\{u \mid u \in E_{l},\|u\| \leq R_{l}\right\},
$$

where $R_{l}>0$ satisfies that $I_{\mu}(u) \leq I(u)<0$ for $u \in E_{l},\|u\| \geq R_{l}$. Define

$$
\begin{aligned}
& c_{l}=\inf _{\varphi \in \Gamma_{l}} \sup _{u \in \varphi\left(B_{l}\right) \backslash W} I_{\mu}(u), \quad l=2,3, \cdots, \\
& \Gamma_{l}=\left\{\varphi \mid \varphi \in C\left(B_{l}, H_{0}^{1}(\Omega)\right), \varphi \text { is odd, }\left.\varphi\right|_{\partial B_{l}}=I d\right\} .
\end{aligned}
$$


Lemma 2.8. There exist $0<\alpha_{l}<\beta_{l}, l=2,3, \cdots$, such that

$$
\alpha_{l} \leq c_{l}(\mu) \leq \beta_{l}, \quad l=2,3, \cdots, \quad \text { and } \quad \alpha_{l} \rightarrow+\infty \quad \text { as } l \rightarrow+\infty .
$$

Proof. Since $I d \in \Gamma_{l}$, we have

$$
c_{l}(\mu) \leq \sup _{B_{l} \backslash W} I_{\mu}(u) \leq \sup _{E_{l}} I(u):=\beta_{l} .
$$

By the condition $\left(f_{1}\right)$, we can choose $\tau>0$ such that

$$
\begin{aligned}
I_{\mu}(u) & \geq \frac{1}{2} \sigma \int_{\Omega}|\nabla u|^{2} d x-\int_{\Omega}\left(\frac{1}{2} \tau u^{2}+\frac{1}{r} c_{\tau}|u|^{r}\right) d x \\
& \geq \frac{1}{4} \sigma \int_{\Omega}|\nabla u|^{2} d x-\frac{1}{r} c_{\tau} \int_{\Omega}|u|^{r} d x:=L(u) .
\end{aligned}
$$

Define

$$
M=\left\{\left.u\left|u \in H_{0}^{1}(\Omega), \frac{\sigma}{2} \int_{\Omega}\right| \nabla u\right|^{2} d x-c_{\tau} \int_{\Omega}|u|^{r} d x \geq 0\right\} .
$$

By choosing $R_{l}$ large enough, we can assume

$$
\partial B_{l} \cap M=\varnothing .
$$

By Lemma 4.5 [16] for $v_{0}$ sufficiently small it holds that

$$
\left(\varphi\left(B_{l}\right) \backslash W\right) \cap \partial M \cap E_{l-2}^{\perp} \neq \varnothing \quad \text { for } \varphi \in \Gamma_{l}, \quad l=2,3, \cdots .
$$

Hence

$$
c_{l}(\mu)=\inf _{\varphi \in \Gamma_{l}} \sup _{u \in \varphi\left(B_{l}\right) \backslash W} I_{\mu}(u) \geq \inf _{u \in \partial M \cap E_{l-2}^{\perp}} I_{\mu}(u) \geq \inf _{u \in \partial M \cap E_{l-2}^{\perp}} L(u) .
$$

We claim that there exist positive constants $\tau$ and $c$, independent of $\mu$ and $l$ such that

$$
c_{l}(\mu) \geq \inf _{u \in \partial M \cap E_{l-2}^{\perp}} L(u) \geq c \lambda_{l-1}^{\tau}:=\alpha_{l}, \quad l=2,3, \cdots .
$$

Choose $p \in\left(r, 2^{*}\right), t \in(0,1)$ such that $\frac{1}{r}=\frac{t}{2}+\frac{1-t}{p}$. For $u \in \partial M \cap E_{l-2}^{\perp}$, we have

$$
\begin{aligned}
\frac{\sigma}{2} \int_{\Omega}|\nabla u|^{2} d x & =c_{\tau} \int_{\Omega}|u|^{r} d x \leq c_{\tau}\left(\int_{\Omega} u^{2} d x\right)^{\frac{r}{2} t}\left(\int_{\Omega}|u|^{p} d x\right)^{\frac{r}{p}(1-t)} \\
& \leq c_{\tau}\left(\lambda_{l-1}^{-1} \int_{\Omega}|\nabla u|^{2} d x\right)^{\frac{r}{2} t} \cdot\left(c_{p} \int_{\Omega}|\nabla u|^{2} d x\right)^{\frac{r}{2}(1-t)} \\
& =c \lambda_{l-1}^{-\frac{r}{2} t}\left(\int_{\Omega}|\nabla u|^{2} d x\right)^{\frac{r}{2}}
\end{aligned}
$$

hence

$$
\begin{aligned}
& \int_{\Omega}|\nabla u|^{2} d x \geq c \lambda_{l-1}^{\frac{r}{r-2} t}, \\
& L(u)=\left(\frac{1}{4}-\frac{1}{2 r}\right) \sigma \int_{\Omega}|\nabla u|^{2} d x \geq c \lambda_{l-1}^{\frac{r}{r-2} t} .
\end{aligned}
$$

Thus, we complete the proof. 
We are in a position to prove Theorem 1.1. To do it, we need the following regularity result for solutions of the quasilinear elliptic equation (2.13), the proof of which will be given in Appendix.

Proposition 2.2. Let $u \in H_{0}^{1}(\Omega), D I_{\mu}(u)=0$ and $I_{\mu}(u) \leq L$. Then there exist $K>0, \gamma \in$ $(0,1)$, depending on L only, such that

$$
\|u\|_{C^{1, \gamma}(\bar{\Omega})} \leq K .
$$

Proof of Theorem 1.1. Given an integer $l \geq 2$, by Proposition 2.1 there exist $u_{l}(\mu) \in H_{0}^{1}(\Omega)$, $\mu \in(0,1]$ such that $u_{l}(\mu)$ is sign-changing, $D I_{\mu}\left(u_{l}(\mu)\right)=0, \alpha_{l} \leq I_{\mu}\left(u_{l}(\mu)\right) \leq \beta_{l}$. By Proposition 2.2 there exist $k_{l}=k_{l}\left(\beta_{l}\right)>0, \gamma_{l}=\gamma_{l}\left(\beta_{l}\right) \in(0,1)$ such that $\left\|u_{l}(\mu)\right\|_{C^{1, \gamma_{l}}(\bar{\Omega})} \leq$ $k_{l}$. Choose $\mu_{l}$ such that $b_{+} k_{l}^{2} \leq \frac{1}{2 \mu_{l}}$. Denote $u_{l}=u_{l}\left(\mu_{l}\right)$, then

$$
\begin{array}{ll}
\sum_{i, j=1}^{N} \beta_{i j}\left(u_{l}\right) D_{i} u_{l} D_{j} u_{l} \leq b_{+} k_{l}^{2} \leq \frac{1}{2 \mu_{l}} & \text { for } x \in \bar{\Omega}, \\
h_{\mu}^{\prime}\left(\sum_{i, j=1}^{N} \beta_{i j}\left(u_{l}\right) D_{i} u_{l} D_{j} u_{l}\right) \equiv 1 & \text { for } x \in \bar{\Omega} .
\end{array}
$$

$u_{l}$ is a sign-changing solution of the original equation (1.1), and $I\left(u_{l}\right)=I_{\mu_{l}}\left(u_{l}\right)=c_{l}\left(\mu_{l}\right) \geq$ $\alpha_{l} \rightarrow \infty$ as $l \rightarrow+\infty$. We obtain infinitely many sign-changing solutions of Eq. (1.1)

Remark 2.1. Without the assumptions $\left(b_{4}\right),\left(f_{4}\right)$, we have the following theorem of three nontrivial solutions, which is reminiscent of the well known result for semilinear elliptic equations such as in $[7,9,23,25]$.

Theorem 2.1. Assume $\left(b_{1}\right)-\left(b_{3}\right),\left(f_{1}\right)-\left(f_{3}\right)$. Then Eq. (1.1) has at least three nontrivial solutions, one is positive, one is negative and the third is sign-changing.

Again we apply the method of invariant sets of the descending flow, see $[6,18,19]$. We leave the detail of proof to the interested readers.

\section{Appendix}

Proposition A.1. Assume $u \in H_{0}^{1}(\Omega), D I_{\mu}(u)=0$ and $I_{\mu}(u) \leq L$. Then there exist $K>0$, $\gamma \in(0,1)$, depending on L only, such that

$$
\|u\|_{C^{1, \gamma}(\bar{\Omega})} \leq K .
$$

First we apply Moser's iteration to obtain the $L^{\infty}$-bound.

Lemma A.1. Assume $u \in H_{0}^{1}(\Omega), D I_{\mu}(u)=0$ and $I_{\mu}(u) \leq L$. Then there exists $M>0$, depending on L only, such that

$$
|u|_{L^{\infty}(\Omega)} \leq M
$$


Proof. First we note that $u$ satisfies the equation

$$
\begin{aligned}
& \sigma \int_{\Omega} \nabla u \nabla \varphi d x+\int_{\Omega} h_{\mu}^{\prime}\left(\sum_{i, j=1}^{N} \beta_{i j}(u) D_{i} u D_{j} u\right) \sum_{i, j=1}^{N}\left(\beta_{i j}(u) D_{i} u D_{j} \varphi\right. \\
& \left.\quad+\frac{1}{2} D_{z} \beta_{i j}(u) D_{i} u D_{j} u \varphi\right) d x \\
& =\int_{\Omega} f(u) \varphi d x
\end{aligned}
$$

for $\varphi \in H_{0}^{1}(\Omega)$. Given $T>0$. Let $u^{T}$ be the truncated function: $u^{T}=u$ if $|u| \leq T$; $u^{T}= \pm T$ if $\pm u \geq T$. Assume $s>1$, take $\varphi=\left|u^{T}\right|^{2 s-2} u$ as test function in (A.2). Notice that

$$
\int_{\Omega} h_{\mu}^{\prime}\left(\sum_{i, j=1}^{N} \beta_{i j}(u) D_{i} u D_{j} u\right) \sum_{i, j=1}^{N}\left(\beta_{i j}(u) D_{i} u D_{j} \varphi+\frac{1}{2} D_{z} \beta_{i j}(u) D_{i} u D_{j} u \varphi\right) d x \geq 0,
$$

we have

$$
\sigma \int_{\Omega} \nabla u \nabla \varphi d x \leq \int_{\Omega} f(u) \varphi d x
$$

Choose $p=\frac{2 N}{N-2}$ if $N \geq 3 ; p \in(r,+\infty)$ if $N=1,2$. By the Sobolev embedding theorem and (2.3) in Lemma 2.3,

$$
|u|_{L^{\gamma}(\Omega)} \leq c\|u\| \leq c(L) .
$$

Denote $d=\frac{2+p-r}{2}, d>1$. Assume

$$
\int_{\Omega}|u|^{s p \cdot \frac{1}{d}} d x<+\infty
$$

Let $T \rightarrow \infty$ in (A.3), we obtain

$$
(2 s-1) \sigma \int_{\Omega}|\nabla u|^{2}|u|^{2 s-2} d x \leq \int_{\Omega}|f(u)||u|^{2 s-1} d x .
$$

The left hand side of (A.4),

$$
\text { LHS } \geq\left.\left.\frac{c}{s} \int_{\Omega}|\nabla| u\right|^{s-1} u\right|^{2} d x \geq \frac{c}{s}\left(\int_{\Omega}|u|^{s p} d x\right)^{\frac{2}{p}} .
$$

The right hand side of (A.4),

$$
\begin{aligned}
\text { RHS } & \leq \int_{\Omega} c\left(1+|u|^{r-1}\right)|u|^{2 s-1} d x \leq c\left(1+\int_{\Omega}|u|^{r-2} \cdot|u|^{2 s} d x\right) \\
& \leq c\left(1+\left(\int_{\Omega}|u|^{p} d x\right)^{\frac{r-2}{p}}\left(\int_{\Omega}|u|^{s p \cdot \frac{2}{2+p-r}} d x\right)^{\frac{2+p-r}{p}}\right) \\
& \leq c\left(1+\left(\int_{\Omega}|u|^{s p \cdot \frac{1}{d}} d x\right)^{\frac{2 d}{p}}\right) \\
& \leq c \max \left\{1,\left(\int_{\Omega}|u|^{s p \cdot \frac{1}{d}} d x\right)^{\frac{2 d}{p}}\right\} .
\end{aligned}
$$


By (A.5) and (A.6), we have

$$
\max \left\{1,\left(\int_{\Omega}|u|^{s p} d x\right)^{\frac{1}{s p}}\right\} \leq(c s)^{\frac{1}{2 s}} \max \left\{1,\left(\int_{\Omega}|u|^{s p \cdot \frac{1}{d}} d x\right)^{\frac{d}{s p}}\right\} .
$$

Starting from $s_{0}=d>1$, by iteration we obtain

$$
|u|_{L^{\infty}(\Omega)} \leq c\left(1+|u|_{L^{p}(\Omega)}\right) \leq c(1+\|u\|) \leq C(L) .
$$

Thus, we complete the proof.

Proof of Proposition A.1. We write down the quasilinear equation (1.1) in the divergence form

$$
\begin{cases}Q(u)=\operatorname{div} A_{\mu}(u, \nabla u)+B_{\mu}(u, \nabla u)=0 & \text { in } \Omega, \\ u=0 & \text { on } \partial \Omega,\end{cases}
$$

where $A_{\mu}(z, p)=\left(A_{\mu}^{i}(z, p)\right)_{i=1}^{N}, z \in \mathbb{R}, p=\left(p_{i}\right) \in \mathbb{R}^{N}$ and

$$
\begin{aligned}
& A_{\mu}^{i}(z, p)=\sigma p_{i}+h_{\mu}^{\prime}\left(\sum_{i, j=1}^{N} \beta_{i j}(z) p_{i} p_{j}\right) \sum_{i, j=1}^{N} \beta_{i j}(z) p_{j}, \quad i=1, \cdots, N, \\
& B_{\mu}(z, p)=-h_{\mu}^{\prime}\left(\sum_{i, j=1}^{N} \beta_{i j}(z) p_{i} p_{j}\right) \sum_{i, j=1}^{N} D_{z} \beta_{i j}(z) p_{i} p_{j}+f(z) .
\end{aligned}
$$

Denote

$$
\begin{aligned}
a_{\mu}^{i j}=\frac{\partial A_{\mu}^{i}}{\partial p_{j}}=\sigma \delta_{i j} & +h_{\mu}^{\prime}\left(\sum_{i, j=1}^{N} \beta_{i j}(z) p_{i} p_{j}\right) \beta_{i j}(z) \\
& +2 h_{\mu}^{\prime \prime}\left(\sum_{i, j=1}^{N} \beta_{i j}(z) p_{i} p_{j}\right) \sum_{i=1}^{N} \beta_{i j}(z) p_{i} \sum_{j=1}^{N} \beta_{i j}(z) p_{j}
\end{aligned}
$$

for $i, j=1, \cdots, N$. We verify the structure conditions satisfied by the quasilinear equation $(Q)$ by use of the assumptions $(B),(F)$ and the property of the truncated function $h_{\mu}$.

$$
\begin{aligned}
& \left(a_{1}\right)\left|A_{\mu}(z, p)\right| \leq \sigma|p|+\left|\left(\sum_{j=1}^{N} \beta_{i j}(z) p_{j}\right)_{i=1}^{N}\right| \leq \sigma|p|+\left(b_{+}-\sigma\right)|p|=b_{+}|p| . \\
& \left(a_{2}\right) p \cdot A_{\mu}(z, p)=\sigma|p|^{2}+h_{\mu}^{\prime}\left(\sum_{i, j=1}^{N} \beta_{i j}(z) p_{i} p_{j}\right) \sum_{i, j=1}^{N} \beta_{i j}(z) p_{i} p_{j} \geq \sigma|p|^{2} . \\
& \text { (a) } \quad \sum_{i, j=1}^{N} a_{\mu}^{i j}(z, p) \xi_{i} \xi_{j}
\end{aligned}
$$




$$
\begin{aligned}
& =\sigma|\xi|^{2}+h_{\mu}^{\prime}\left(\sum_{i, j=1}^{N} \beta_{i j}(z) p_{i} p_{j}\right) \sum_{i, j=1}^{N} \beta_{i j}(z) \xi_{i} \xi_{j}+2 h_{\mu}^{\prime \prime}\left(\sum_{i, j=1}^{N} \beta_{i j}(z) p_{i} p_{j}\right)\left(\sum_{i, j=1}^{N} \beta_{i j}(z) p_{i} \xi_{j}\right)^{2} \\
& \geq \sigma|\xi|^{2}-2\left|h_{\mu}^{\prime \prime}\left(\sum_{i, j=1}^{N} \beta_{i j}(z) p_{i} p_{j}\right)\right| \cdot \sum_{i, j=1}^{N} \beta_{i j}(z) p_{i} p_{j} \cdot \sum_{i, j=1}^{N} \beta_{i j}(z) \xi_{i} \xi_{j} \\
& \geq \sigma|\xi|^{2}-2 \varepsilon b_{+}|\xi|^{2} \geq \frac{1}{2} \sigma|\xi|^{2} \text { for } \xi=\left(\xi_{i}\right) \in \mathbb{R}^{N} .
\end{aligned}
$$

$\left(a_{4}\right)$ For $z, w \in \mathbb{R}$, denote $y_{t}=t z+(1-t) w, t \in(0,1]$. Then

$$
\begin{aligned}
& \left|A_{\mu}^{i}(z, p)-A_{\mu}^{i}(w, p)\right| \\
= & \left|\int_{0}^{1} d_{t}\left\{h_{\mu}^{\prime}\left(\sum_{i, j=1}^{N} \beta_{i j}\left(y_{t}\right) p_{i} p_{j}\right) \sum_{i, j=1}^{N} \beta_{i j}\left(y_{t}\right) p_{j}\right\}\right| \\
\leq & \left|\int_{0}^{1} h_{\mu}^{\prime}\left(\sum_{i, j=1}^{N} \beta_{i j}(z) p_{i} p_{j}\right) \sum_{i, j=1}^{N} D_{z} \beta_{i j}\left(y_{t}\right) p_{j}(z-w) d t\right| \\
& \quad+\left|\int_{0}^{1} h_{\mu}^{\prime \prime}\left(\sum_{i, j=1}^{N} \beta_{i j} p_{i} p_{j}\right) \sum_{i, j=1}^{N} D_{z} \beta_{i j}\left(y_{t}\right) p_{i} p_{j}(z-w) \cdot \sum_{j=1}^{N} \beta_{i j}\left(y_{t}\right) p_{j} d t\right| \\
\leq & c|p||z-w| .
\end{aligned}
$$

(b) On the other hand, we have

$$
\begin{aligned}
\left|B_{\mu}(z, p)\right| & \leq\left|h_{\mu}^{\prime}\left(\sum_{i, j=1}^{N} \beta_{i j}(z) p_{i} p_{j}\right) \sum_{i, j=1}^{N} D_{z} \beta_{i j}(z) p_{i} p_{j}\right|+|f(z)| \\
& \leq c|p|^{2}+\Lambda(M) \leq \Lambda(M)\left(1+|p|^{2}\right) \quad \text { for } z \in \mathbb{R}, \quad|z| \leq M,
\end{aligned}
$$

where $\Lambda(M)$ is an increasing function from $\mathbb{R}^{+}$to $\mathbb{R}^{+}$.

Assume $u \in H_{0}^{1}(\Omega), D I_{\mu}(u)=0, I_{\mu}(u) \leq L$. By Lemma A.1, there exists $M=M(L)$ such that $|u|_{L^{\infty}(\Omega)} \leq M$. The quasilinear equation $(Q)$ satisfies the natural structure conditions for elliptic equation. All the assumptions of Corollary 1.5, Theorem 1.7 of [14] (see also [12]) are fulfilled. Therefore there exist $K>0, \gamma \in(0,1)$ depending only on $M$, $\Lambda$ such that

We complete the proof.

$$
\|u\|_{C^{1, \gamma}(\bar{\Omega})} \leq K
$$

\section{Acknowledgements}

The authors would like to thank the referee for carefully reading the paper and for helpful suggestions. The work is partially supported by NSFC (Nos. 11761082, 11671364, 11771324 and 11831009). 


\section{References}

[1] A. Ambrosetti, and P. H. Rabinowitz, Dual variational methods in critical point theory and applications, J. Funct. Anal., 14 (1973), 349-381.

[2] D. Arcoya and L. Boccardo, Some remarks on critical point theory for nondifferentiable functionals, Nonl. Diff. Equa. Appl., 6 (1999), 79-100.

[3] D. Arcoya and L. Boccardo, and L. Orsina, Existence of critical points for some noncoercive functionals, Ann. Inst. H. Poincaré Anal. Non Linéaire, 18 (2001), 437-457.

[4] T. Bartsch, Critical point theory on partially ordered Hilbert spaces, J. Funct. Anal., 186 (2001), 117-152.

[5] T. Bartsch, K.-C. Chang, and Z.-Q. Wang, On the Morse indices of sign-changing solutions for nonlinear elliptic problems, Mathematische Zeitschrift, 233 (2000), 655-677.

[6] T. Bartsch, Z. Liu, and T. Weth, Nodal solutions of a $p$-Laplacian equation, Proc. London Math. Soc., 91 (2005), 129-152.

[7] T. Bartsch, and Z.-Q. Wang, On the existence of sign changing solutions for semilinear Dirichlet problems, Topol. Methods Nonlinear Anal., 7 (1996), 115-131.

[8] A. Canino, and M. Degiovanni, Nonsmooth critical point theory and quasilinear elliptic equations, in: A. Granas, M. Frigon and G. Sabidussi (eds), Topological Methods in Differential Equations and Inclusions, NATO ASI Series (Series C: Mathematical and Physical Sciences), vol 472, Kluwer, Dordrecht, (1995), 1-50.

[9] E. N. Dancer, and Y. Du, A note on multiple solutions of some semilinear elliptic problems, J. Math. Anal. Appl., 211 (1997), 626-640.

[10] A. Ioffe, and E. Schwartzman, Metric critical point theory 1, Morse regularity and homotopic stability of a minimum, J. Math. Pures Appl., 75 (1996), 125-153.

[11] G. Katriel, Mountain pass theorems and global homeomorphism theorems, Ann. Inst. H. Poincaré Anal. Non Linéaire, 11 (1994), 189-209.

[12] O. A. Ladyzhenskaya, and N. N. Ural'tseva, Linear and quasilinear elliptic equations. Translated from the Russian by Scripta Technica, Inc. Translation editor: Leon Ehrenpreis Academic Press, New York-London, 1968.

[13] S. Li, and Z.-Q. Wang, Lusternik-Schnirelman theory in partially ordered Hilbert spaces, Trans. Amer. Math. Soc., 354 (2002), 3207-3227.

[14] G. M. Lieberman, The natural generalization of the natural conditions of Ladyzhenskaya and Ural'tseva for elliptic equations, Commun. Partial Differential Equations, 16 (1991), 311361.

[15] J. Q. Liu, and Y. Guo, Critical point theory for nonsmooth functionals, Nonlinear Anal., 66 (2007), 2731-2741.

[16] J. Q. Liu, X. Q. Liu, and Z.-Q. Wang, Multiple sign-changing solutions for quasilinear elliptic equations via perturbation method, Commun. Partial Differential Equations, 39 (2014), 2216-2239.

[17] J. Q. Liu, X. Q. Liu, and Z.-Q. Wang, Quasilinear equations via elliptic regularization method, Adv. Nonlinear Study, 13 (2013), 517-531.

[18] J. Q. Liu, X. Q. Liu, and Z.-Q. Wang, Multiple mixed states of nodal solutions for nonlinear Schrödinger systems, Calc. Var. Partial Differential Equations, 52 (2015), 565-586.

[19] J. Q. Liu, X. Q. Liu, and Z.-Q. Wang, Sign-changing solutions for coupled nonlinear Schrödinger equations with critical growth, J. Differential Equations, 261 (2016), 7194-7236.

[20] X. Q. Liu, J. Q. Liu, and Z.-Q. Wang, Quasilinear elliptic equations via perturbation method, Proc. Amer. Math. Soc., 141(1) (2013), 253-263. 
[21] X. Q. Liu, J. Q. Liu, and Z.-Q. Wang, Quasilinear elliptic equations with critical growth via perturbation method, J. Differential Equations, 254 (2013), 102-124.

[22] X. Q. Liu, J. Q. Liu, and Z.-Q. Wang, Localized nodal solutions for quasilinear Schrödinger equations, J. Differential Equations, 267 (2019), 7411-7461.

[23] Z. Liu, and J. Sun, Invariant sets of descending flow in critical point theory with applications to nonlinear differential equations, J. Differential Equations, 172 (2001), 257-299.

[24] P. H. Rabinowitz, Minimax Methods in Critical Point Theory with Applications to Differential Equations, CBMS Regional Conf. Ser. in Math., vol. 65, American Mathematical Society, Providence, 1986.

[25] Z.-Q. Wang, On a superlinear elliptic equation, Ann. Inst. H. Poincaré Anal. Non Lin3́aire, 8 (1991), 43-57. 